\title{
Pedestrian Network Data Collection through Location-Based Social Networks
}

\author{
Piyawan Kasemsuppakorn, Hassan A. Karimi \\ Geoinformatics Laboratory, School of Information Sciences \\ University of Pittsburgh, Pittsburgh, PA, USA \\ Email: pik2@pitt.edu, hkarimi@mail.sis.pitt.edu
}

\begin{abstract}
The increasing capabilities of mobile devices and their mobile positioning technologies have shown great promise in location-enabled applications such as navigation systems. One of the essential components of a navigation system is a spatial database as it provides the base data to perform navigation and routing functions, among others. With the popularity of vehicle navigation systems, road network databases are now well developed and well suited for vehicles traveling on roads. However, road networks are not suitable for pedestrian navigation systems as pedestrians do not walk along the middle of street lanes and are not constrained by the boundaries of the road. Consequently, pedestrian network data is needed in location-enabled applications for pedestrians and other applications including transportation planning and physical activities study. Due to the lack of availability of pedestrian network data, new approaches for acquiring and maintaining pedestrian network data that are efficient and cost effective are needed. This paper presents a new approach that is based on location-based social networking for collecting pedestrian network data. The proposed approach stems from the concept of collaborative mapping where pedestrian network data can be collected by members of a social network recording GPS trajectories. A prototype based on a framework called Social Navigation Network (SoNavNet) for sharing and recommending navigation related information is also discussed.
\end{abstract}

Index Terms-Pedestrian Network, Collaborative Mapping, Social Network, Location-Awareness, Mobility.

\section{INTRODUCTION}

$\mathrm{T}$ he availability of ubiquitous computing devices, wireless networks, and the high accuracy satellite-based positioning systems has paved the way for the development of Location-Based Services (LBS) which provide information relevant to the location of mobile users [1]. These services have changed the way people live and work by allowing mobile users to receive digitized content and services anytime and anywhere. Consequently, many service providers have increased their business opportunities by creating innovative services such as mobile guides and navigation, shopping assistants, emergency services, and social networking [2]. A navigation system, one type of LBS, aims to guide people along a route in order to reach a predetermined destination. One of the essential components for a navigation system is a spatial database [3-4] since it provides the base data to perform navigation and routing functions and to display a map for presenting end-user's location and locations of other spatial objects such as points of interests. With the popularity of vehicle navigation systems, road network databases are now well developed and widely available for many countries in North America, Europe, and Asia. Data sources for road network databases are provided by government agencies, e.g., the U.S. Census Bureau's Topographically Integrated Geographic Encoding and Reference (TIGER), non-profit organizations, e.g., Pennsylvania Spatial Data Access (PASDA), and commercial mapping companies, e.g., NAVTEQ. Currently, pedestrian navigation systems are based on vehicle navigation systems at the data level [5] due to the lack of availability of pedestrian network data. By "pedestrian network" we mean spatial and non-spatial data for sidewalks, crosswalks, footpaths, accessible entrances, pedestrian underpasses, pedestrian overpasses, steps, and trails. Although pedestrian networks and road networks overlap in terms of data content, the two are dissimilar in scale and details. Unlike vehicles, pedestrians do not move along the middle of street lanes and are not constrained by the boundaries of the road. For these reasons, road networks are not appropriate for pedestrian navigation systems [6-7]. A pedestrian navigation system which uses a road network as a substitute for a pedestrian network might suggest a route missing sidewalks or pedestrian paths. To this problem, in many pedestrian navigation prototypes, pedestrian data for testing is manually collected and constructed in selected areas [8-11]. Generally, the most common approach for constructing pedestrian network data is to manually digitize the pedestrian path centerlines by using satellite images, e.g., see [7]. Besides pedestrian navigation, pedestrian networks are used in other applications such as measuring pedestrian accessibility in urban planning. Chin et al., [12] studied the impact of missing pedestrian data on the analysis of pedestrian accessibility, connectivity, and walkability. The results showed that using a pedestrian network offers a more realistic means of measuring the level of pedestrian accessibility than a street network. As current approaches for acquiring and maintaining pedestrian network data, by government agencies or private companies, incur huge investments of time and money, new approaches that are efficient and cost effective are needed.

Web 2.0, the second generation in the web's evolution, facilitates collaborative content and modification, enables the creation of applications by reusing and combining different sources, and establishes connection among people with common interests [13]. An appropriate Web 2.0 technology that can assist in pedestrian data collection is a social network. This coupled with the availability of GPS-enabled mobile phones, portable digital maps, and free web mapping 
platforms, such as Google or Microsoft, provides users with easy access to location information and enables them to also supply their own location content. Such trends have facilitated the feasibility of collaborative mapping which is "an initiative to collectively create models of real-world locations online that anyone can access and use to virtually annotate locations in space" [14]. GPS-Tracks, OpenStreetMap, and Wikimapia are examples of collaborative mapping that allow people to add their own GPS trajectories collected by car or bicycle. The benefit of collaborative mapping is that it allows for a costeffective means of collecting potentially a large of amount location data. An alternative approach based on the concept of collaborative mapping, which we propose, is to utilize a location-based social network (LBSN) to collect and update pedestrian networks. To the best of the authors' knowledge, this is the first attempt to collect pedestrian network data through LBSN. In this paper, we focus on the collection of real-world pedestrian paths through GPS trajectories in a social network prototype based on a framework called Social Navigation Networks (SoNavNet) [15].

Contributions of this paper include analysis and categorization of pedestrian path components and a new approach to collect GPS trajectories for the automatic construction of pedestrian network base maps. The structure of this paper is as follows. Section 2 provides the background on pedestrian networks, a short overview on collaborative mapping, and a brief overview of location tracking technology. This is followed by Section 3 where the LBSN approach is discussed. Section 4 discusses experimentation for collecting pedestrian data through our prototype. Finally, conclusions and future research are discussed in Section 5.

\section{BACKGROUND}

\section{A. Pedestrian Network}

This section will begin by establishing and clarifying terminology used throughout this paper. A "pedestrian" is "any person who is afoot or who is using a wheelchair or a means of conveyance propelled by human power other than a bicycle" [16]. A "pedestrian path" refers to any pathway that is designed for a pedestrian in order to improve pedestrian safety, reduce potential accidents, and promote mobility and accessibility. Most people only consider sidewalks as part of the pedestrian paths, but pedestrian paths can be considered to encompass more. We have identified eight types of pedestrian paths as shown in Table I.

A pedestrian network is a topological map that delineates the geometric relationship between pedestrian path elements generally represented by points and lines. Not only should the network represent the geometry and topology, but it also should contain attribute information about those paths such as path segment length. Unlike road networks, pedestrian networks are found mostly in urban areas and in some areas (e.g., a city) there may be more than one network. A pedestrian network can be modeled as a graph and stored in a spatial database system which supports spatial functions. In
TABLE I

PedestRIAN PATH TyPeS

\begin{tabular}{|c|c|}
\hline Types & Description \\
\hline 1. Sidewalk & $\begin{array}{l}\text { A path designed for pedestrian traffic alongside a } \\
\text { road }\end{array}$ \\
\hline $\begin{array}{l}\text { 2. Pedestrian } \\
\text { Walkway }\end{array}$ & $\begin{array}{l}\text { A path not necessary at the side of a road such as a } \\
\text { walkway between buildings, or a foot path to the } \\
\text { plaza }\end{array}$ \\
\hline $\begin{array}{l}\text { 3. Accessible } \\
\text { Path/Ramp }\end{array}$ & $\begin{array}{l}\text { A part of pedestrian walkway, but specifically at } \\
\text { the entrance of the buildings or ramp for disabled } \\
\text { group }\end{array}$ \\
\hline 4. Crosswalk & $\begin{array}{l}\text { A facility that is marked off on a road to indicate } \\
\text { where pedestrians should cross, generally at an } \\
\text { intersection }\end{array}$ \\
\hline $\begin{array}{l}\text { 5. Pedestrian } \\
\text { Bridges }\end{array}$ & $\begin{array}{l}\text { A grade-separated crossing that is constructed over } \\
\text { the roadway }\end{array}$ \\
\hline $\begin{array}{l}\text { 6. Pedestrian } \\
\text { Tunnels }\end{array}$ & $\begin{array}{l}\text { A grade-separated crossing that is an below- } \\
\text { ground passageway }\end{array}$ \\
\hline 7. Steps & $\begin{array}{l}\text { A grade-separated crossing that is filling the gap in } \\
\text { elevation }\end{array}$ \\
\hline 8. Walking Trails & $\begin{array}{l}\text { A path that is mostly designated as recreational } \\
\text { such as running trails or natural trails }\end{array}$ \\
\hline
\end{tabular}

such a database, a point represents the location of an object, such as an accessible ramp or a decision point, whereas a line represented by a series of points, is used for representing a path, such as a sidewalk or crosswalk. Assigned to each path is a scalar value which in most cases represents segment lengths or sometimes impedance scores such as for a wheelchair users [9]. An example of a pedestrian network is shown in Fig. 1.

Pedestrian navigation systems are not the only application requiring pedestrian networks. Other applications include transportation planning and physical activity studies. The network itself is the foundation for the core operations of a navigation system such as routing. For physical activity studies, the pedestrian network is used as a base map to measure the link node ratio (LNR) or pedestrian route directness (PRD) which indicates the network connectivity. Further information about LNR and PRD for physical activity studies can be found in [12].

\section{B. Collaborative Mapping}

The impetus for collaborative mapping is driven by the lack of publicly available geographic data and the rapid development of mobile positioning technology, locationawareness, and online communities [14]. First, the lack of

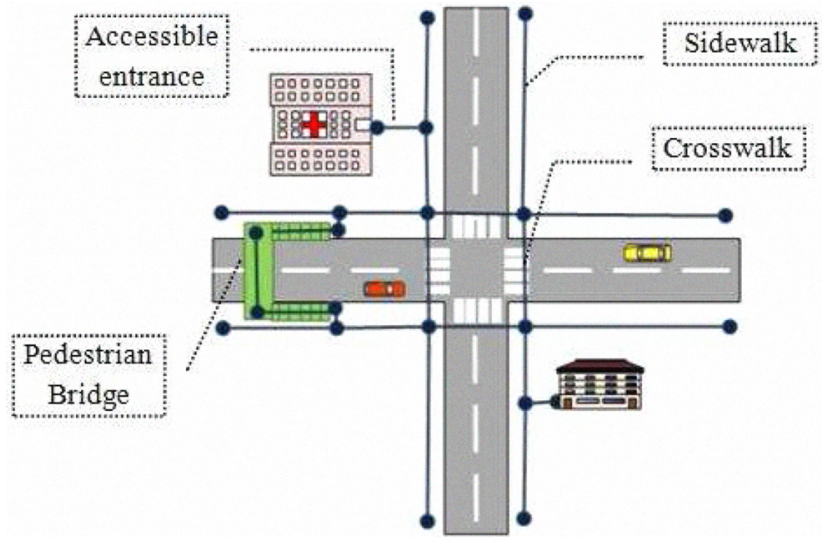

Fig. 1. Example of a Pedestrian Network 
available data is a strong motivation for users to create their own content. The US Geological Survey (USGS) and the Census Bureau provide free or low-cost large-scale geographic data in the United States. Although these are good sources for obtaining data such as road networks, they generally do not provide pedestrian network data. Also, geographic data in other countries or commercial mapping companies is more costly and is subject to strict copyright laws making it a less than ideal data source. Secondly, current mobile devices are equipped with mobile positioning technology, such as network-based technology and GPS, and are available at a reasonable price. These devices provide real-time positioning with relatively good accuracy and precision. Thus, advanced technologies provide individuals with easy access to devices capable of recording and sharing geographic data. Thirdly, with the support of positioning technologies, an emergent technology called location-awareness delivers information from location-aware devices such as GPS-enabled mobile phones to other users or applications, especially LBS applications. Lastly, social networking is an ideal technology that facilitates social connection and coordination among friends and can be integrated with location-enabled applications. Considering the success of social networking and the rapid adoption of mobile devices by a wide variety of users, it is feasible to conceive a mobile social network where members, among other activities, participate in collecting pedestrian network data.

The collaborative mapping process consists of four iterative steps: (1) collection, (2) editing, (3) annotation, and (4) distribution [14]. The first step involves collection of GPS trajectories, which may require a large number of community members to travel a particular area with a GPS-enabled device and make their GPS trajectories available online for other interested members. By GPS trajectories we mean the series of raw GPS data received by mobile devices equipped with GPS receivers. OpenStreetMap (OSM) [17], a community mapping project, provides a tool that allows registered users to create their own contents and upload GPX (GPS eXchange format) trajectories from hand-held GPS units. The OSM community usually organizes a local workshop called "mapping party" which aims to introduce new contributors with the practical experience of collecting and uploading their own contents for a given geographical area to the OSM database. In addition to hand-held GPS units for data collection, we plan to employ recent mobile devices and positioning techniques to record real-time GPS trajectories through LBSN. The second step involves creation of a base map from raw GPS trajectories derived from the first step. Research in this area focuses on updating a road network from GPS trajectories. For example, a map refinement process [18] aims to create a traversable road network from GPS trajectories. Another research project uses an artificial intelligence clustering technique [19] to integrate different GPS trajectories to infer road geometry. The third step of the collaborative mapping process involves providing information on the geometry of the map, called annotation. Examples annotations include a location's name or geotags such as comments on a comfortable path for people with special needs. Annotations make the geographic information more valuable for various applications. The final collaborative mapping step involves making the map available to users. In this paper, we focus only on the first step, collection of pedestrian network base map data.

\section{Location Tracking Technology}

There are various means of location detection on mobile devices such as Cell ID location tracking, GPS, and Wi-Fi positioning systems (WPS). The Cell ID location tracking, or GSM tracing, has been shown to be sufficiently accurate in urban areas where a number of cell phone towers are dense [20]. In general, GPS provides better accuracy than GSM in an open sky environment but does not work well indoors and usually introduces a startup delay for acquiring satellite signals. WPS, pioneered by Skyhook Wireless, uses 802.11 radio signals emitted from wireless routers to determine the location of any Wi-Fi enabled device such as a laptop, PDA, or mobile phone with fairly accurate results. Skyhook has built and maintained a large databases of Wi-Fi access points that cover major cities in North America, Europe, and Asia [21]. However, WPS does not work well when there is no wireless signal. With these shortcomings, the hybrid positioning system which combines WPS, GPS, and GSM to determine more accurate position is introduced such as Skyhook's hybrid positioning system (XPS) [22]. Nevertheless, this positioning methodology requires more computation power which subsequently decreases the battery life of mobile devices.

One aspect of our research focuses on investigating the capability of GPS on mobile devices to estimate the actual position while walking because the quality of raw GPS trajectories is essential in generating pedestrian network base maps. The GPS standard positioning system (SPS) performance specification by the U.S. government is $\pm 9 \mathrm{~m}$ horizontal accuracy 95 percent of the time [23]. However, the GPS accuracy is affected by a number of factors such as noise, atmospheric conditions, natural barriers, and traveling speed. Since GPS devices rely on line of sight of satellites to produce an accurate position, vehicles traveling further away from buildings and tree canopies may be in line of sight and receive higher positioning accuracy than pedestrian or wheelchair who is traveling along the sidewalk. At low speed, estimated positions received from GPS become inaccurate as shown in [24].

\section{LOCATION-BASED SOCIAL NETWORKING}

Social networks or online communities have been around since 1997 with the launch of SixDegrees.com, which allowed users to create profiles, list their friends, surf their friends' lists, and send messages to others [25]. Since then many other social network sites have been launched providing virtual communities where people with similar interests can communicate and share information. Most existing popular social network sites, such as Friendster, Facebook, or hi5, support users by providing tools that facilitate communication with groups of friends. Some of these tools provide users the ability to post personal information, share photos, tag content, 
and link to other users. The development of LBS has further expanded social networking from the standard web-based version to mobile devices and is termed LBSN. A distinctive feature of LBSN on mobile devices is the ability of users to broadcast their own location, search for nearby friends, organize meeting places and activities, and share real-life experiences. Loopt, Rummble, and GyPSii are examples of LBSN. Indeed, there has been a dramatic growth in the number of active users of mobile social network sites with forecasted growth from 54 million in 2008 to nearly 730 million in 2013 [26]. The reason for this can be attributed to people wanting to meet new friends, to get in touch with their networks, to get comments on their uploaded content, and to utilize location services. In addition, research in [27] analyzed the structure of mobile social network and confirmed that it is a typical "scale-free network" that the degree distribution of connectivity almost fits the power law distribution like other social networks.

Our proposed approach is based on the concept of collaborative mapping where the collection of pedestrian networks requires a large group of LBSN members to travel an area to be mapped with a GPS receiver. LBSN are appropriate for collecting pedestrian networks because the scale-free model of social networks provides a very large user base with high connectivity among members and location services on mobile devices provide a cheap and effective solution.

\section{A. Social Networking}

To develop a successful LBSN, it is essential to accurately define goals and identify user motivations. The goal of our proposed LBSN is to facilitate an environment where members of the network can participate in collaborative mapping projects. We focus on developing socialization and content sharing communities in order to accomplish our purpose and to provide the services users are most interested in. The user could be any mobile user who wishes to socialize with others and share digital content, especially location information. An individual can be both a contributor, providing location or other content such as digital media, and a consumer, using the content provided by others. The potential contributors include volunteers and general mobile social network members. We envision data collection by volunteers to be similar to the mapping parties found in the OpenStreetMap project [17] where large groups of people within a given area are offered guidance for collecting realworld data using GPS units. Students in GIS courses are potential volunteers for collecting location as it helps them learn how GPS works and how to collect data with GPS. Although these volunteer communities could positively contribute to generating new pedestrian network data, it is unlikely they will be able to cover all pedestrian paths in a large area such as a city. Other motivations for collecting data could be related to health and leisure services where GPS trajectories are a by-product of their activities. An interesting service supporting health purposes is to allow users to record GPS trajectories along with performance statistics such as distance, speed, and calories burned during exercise or outdoor activities and to share them with friends. A service for leisure purpose is geodiary where people can record GPS trajectories and annotate them with, points of interest. For example, a user goes for a walk collecting data and posting information about the favorite places to walk. Users can keep their trajectories private or publish them to the social network. The incentives of this group of contributors are to enjoy the services from the application, to get community sense by exchanging information with other friends, and to derive personal satisfaction from contributing valuable information, among others.

The assumption is that GPS-enabled mobile phones are not a major problem to obtain as most phones released today have this capability [28] and the LBSN approach imposes certain constraints when collecting location data. Such constraints include the walkable distance, the complexity of the mobile service, the characteristics of an area, and the quality of GPS trajectories. The two former constraints are related to human ability, while the latter two are related to the environment. For the walkable distance, the average human walking distance a day is 1.5 miles in general [29]; therefore, a large number of participants are needed to collect data in large areas. To keep up-to-date pedestrian network data, members should volunteer to participate in data collection when such updates are needed. We believe that the simplicity of the mobile service induces more people to regularly use the services. The characteristics of an area include the density of a city in terms of pedestrian paths. The closer the buildings are together, the narrower the streets are, and the closer the pedestrian footpaths are. Thus, a dense city has a dense pedestrian network and requires more contributors than a sparse city. Lastly, the quality of GPS trajectories is affected by the types of GPS receivers members have available to them and by the accuracy and continuity of GPS signals in some areas.

\section{B. Use Cases}

As mentioned earlier, the collaborative mapping process consists of four steps for generating pedestrian network data. The use case diagram of our location-based social networking approach for data collection purposes is shown in Fig. 2.

We first explain the notation of the diagram as follows. The user symbol represents actors that play different roles in the social network and these roles can be engaged by the same person or multiple people. The ovals represent use cases describing a sequence of actions and the solid lines indicate the associations between actors and use cases. The greyshaded ovals highlight the actions for recording and sharing GPS trajectories. A parent-child relationship between actors is a generalization that defines overlapping roles between actors. A social network member can act as both a contributor and a consumer. The dashed arrows with the label "<<extends $>>$ " depicts an extended relationship between use cases. For example, the Download trajectories are added to the existing use case called view shared information of friends. In simple term, this means that a user usually receives shared information from friends in the network, not from unknown 


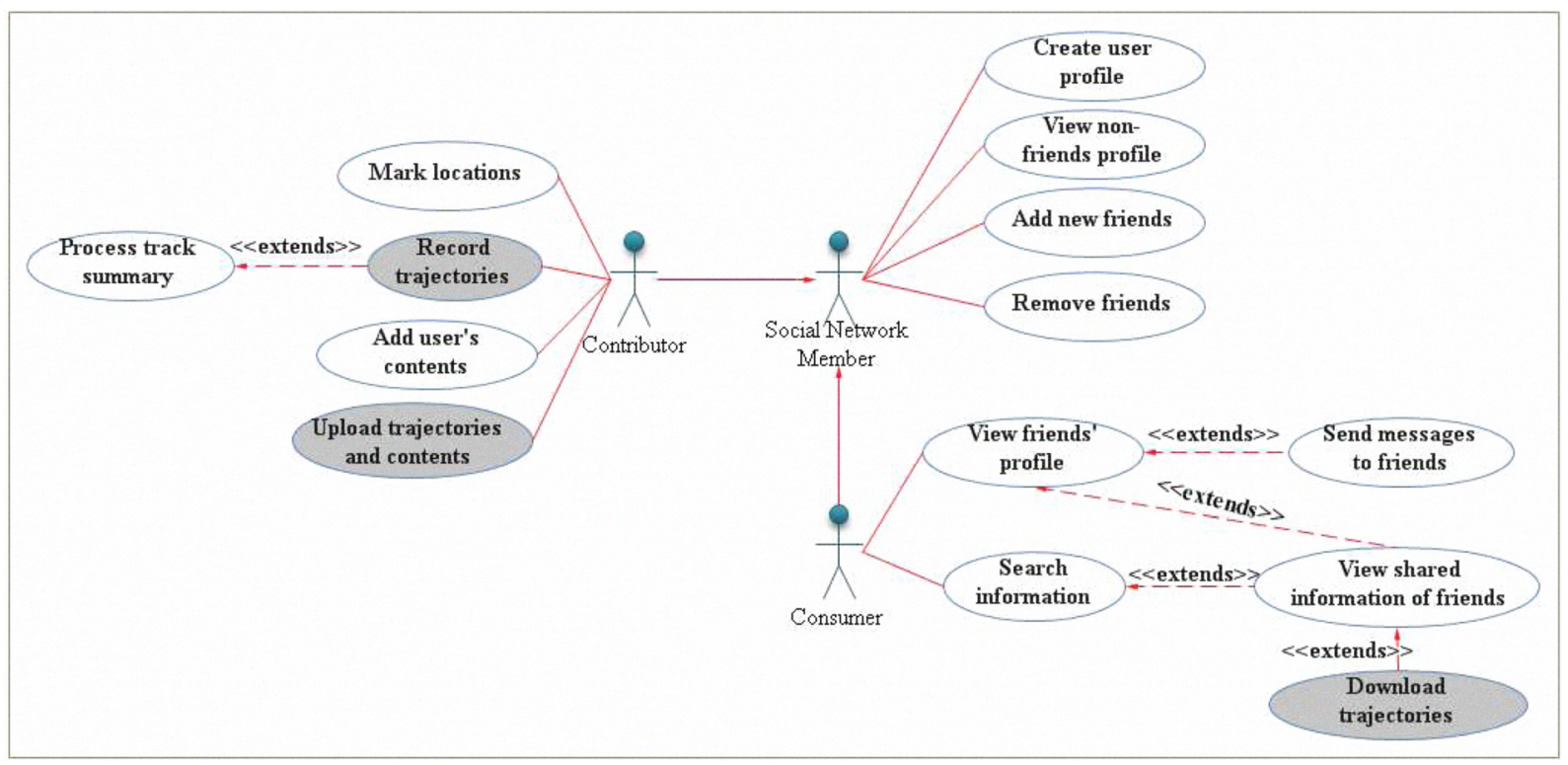

Fig. 2. Use Case Diagram of Location-Based Social Networking

members. As can be seen in the diagram, a member is required to create a user profile in order to become a member which then enables them to establish their own network of contacts by adding and removing other members. The system will be accessible by members from a standard web interface or mobile interface with features corresponding to their method of access. Obviously a user would not be able to record GPS trajectories from their home desktop computer.

In Fig. 2, the use case diagram of the consumer role illustrates how a member might view or download shared spatial and non-spatial data from the system. In this scenario, registered members who would like to obtain location information in a given area can search for shared information posted by friends. Location information includes points of interest, personalized routes between places, and real-world GPS trajectories of trips. Next, they can view or download this location information through the information sharing function of the system. If there is no desired information, they can view friends' profiles and request information by messaging friends that might have data for a specific area.

In Fig. 2, the use case diagram of the contributor role describes how a member creates new content, both spatial and non-spatial, for the system. For example, contributors can use the system for health purposes, where they record GPS trajectories with their performance statistics like speed, elevation, distance, and calories burned during outdoor activities such as walking or running. Another use of the system for travelling or leisure purposes might be for contributors to mark interesting locations and record/annotate trajectories during the course of the trip. Once recorded, users can upload data to the server and make the content available to others or keep it private.

\section{EXPERIMENTATION}

\section{A. Prototype}

A data collection prototype was developed based on the SoNavNet framework. Members of the prototype can create user profiles, establish their own friend lists, record GPS trajectories, and share information with other members for the purpose of collecting a pedestrian network base map.

SoNavNet is a framework for developing navigation-related social networks supporting interaction among both mobile users and web users. Its infrastructure supports application development on a social network that is available to everyone with access to the WWW. Unlike most existing social networks, SoNavNet is focused on navigation in which user mobility is a key issue. Its functions include common social networking activities along with spatially-oriented activities. Users are able to create user profiles, establish relationships with other members, and keep in touch with friends through common social networking functions. The spatial functions include locations such as points of interests, routing, and boundary of designated area. Users can annotate any locations, routes, or geofenced areas of interest on a map with personalized information such as noting a particular route as the most scenic for a running trail. Location tracking is another spatial function that records user's real-time trajectories as they travel along the walking trips. SoNavNet also acts as an information center that allows users to share information about a particular area such as recommendations or GPS trajectories. The location tracking and information sharing functions of SoNavNet are well suited for pedestrian network data collection.

SoNavNet is based on a three-tier architecture: client, middle-tier server, and database server. The client includes mobile users and web users who are able to use the system via a mobile user interface (UI) or web UI. The web server acts as a middle-tier server that provides the core functionalities of 


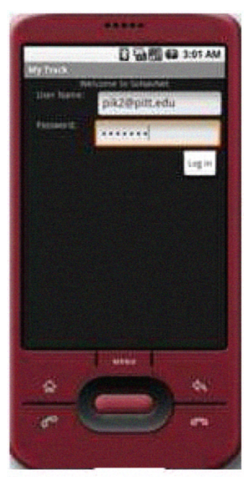

(a)

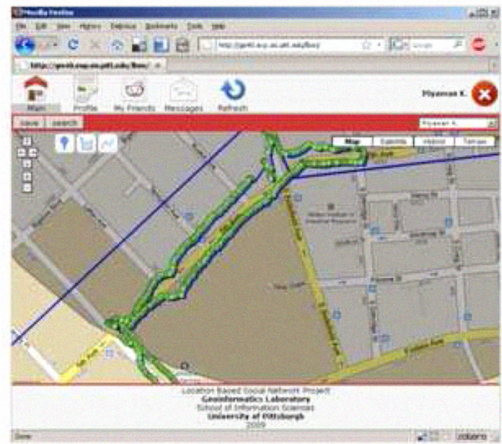

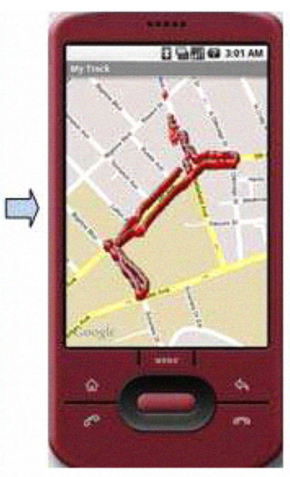

(c)

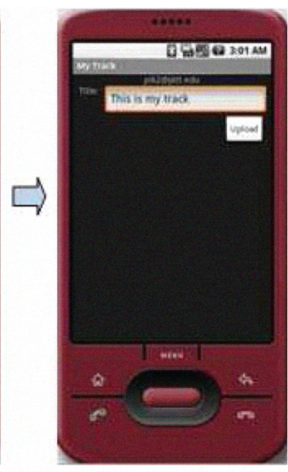

(d)

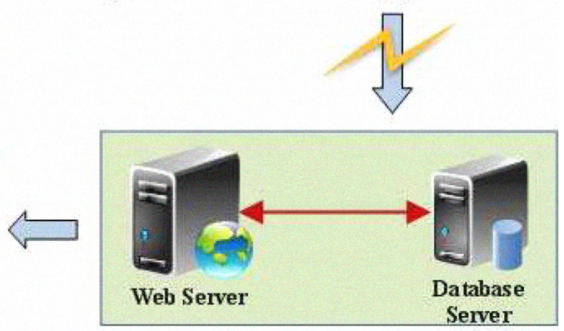

Fig. 3. Screenshots of sidewalk trajectories recording and sharing

the system and is able to request additional services from external service providers such as a map service provider. The database server is responsible for data storage and management. Two types of data are contained in this database: spatial data and non-spatial data. Spatial data includes GPS trajectories, points of interests, and tagged locations. Nonspatial data includes profiles and friends' lists. All information in the database server are kept confidential and revealed only to authorized users. The prototype was developed based on SoNavNet's architecture and was designed for Android-based handsets because of the open platform and development tools available from the Android SDK supported by Google. The prototype has been implemented in Java for the mobile version and in PHP as a scripting language for the web version. The mobile positioning service and the wireless network service are accessed in order to acquire user's current locations and upload contents to the server. For data management, MySQL was used to maintain a database running on a server.

\section{B. Experimentation}

The prototype has been made available to the Geoinformatics Laboratory's members, a group of students as friends and peers with similar interests. The members are able to participate in the social network through a web client or a phone client. The web UI allows users to manage their own profiles and contact lists as well as other functions such as searching and sharing information as discussed previously.

Once SoNavNet is installed on an Android-based phone, users are able to login to the network and have their location drawn on a base map as shown in Fig. $3 a$ and $3 b$, respectively. The updated locations can be received at fixed time intervals (e.g., 1 second) during a trip. As soon as a user has completed their trip and stopped recording, the system will then show recorded locations from start to finish as shown in Fig. 3c. From here the user can annotate the data collected by posting messages or notes as shown in Fig. $3 \mathrm{~d}$. Then the data is ready to upload to the database server. Once uploaded, the annotated GPS trajectory is displayed on the map through the web UI and made available for other members who are interested in and have privileges to download the trajectory.

\section{Data Analysis}

Nine members of the Geoinformatics Laboratory, School of Information Sciences at the University of Pittsburgh, participated in collecting data for testing the prototype and completed a questionnaire expressing their feedback on the data collection experience. The questionnaire consists of two sets of questions: the first set is focused on respondent's background and the second set on usability and experience.

The result from the first part is explained in the following. Of the nine respondents, two-thirds (67\%) were male and onethird $(33 \%)$ were female. All respondents $(100 \%)$ have their own mobile phones and carry their phone with them all the time. Only one-third of the respondents own a smart phone (GPS-enabled and Wi-Fi support). All respondents (100\%) are currently a member of other social networking sites such as Facebook or Hi5. Four of them post and share information (e.g., photos, contents, and videos) with members of those social networking sites several times a week, while the rest of the respondents share information approximately once a month. The result of the feedback in the first part indicates that: (1) carrying a mobile phone when walking outside is not 
considered an extra activity and (2) most people are familiar with social networking and information sharing.

Part 2 of the questionnaire is composed of two sections which are related to usability and experience. We used a Likert scale (1-to-5 rating scale) in order to specify respondents' level of agreement to each question, where 1 is "strongly disagree" and 5 is "strongly agree". The result on usability indicates that of all respondents, $22 \%$ agree and $78 \%$ strongly agree that the data collection process was easy and straightforward, even if they did not know how to collect GPS data. Six of them indicated that they were able to collect the required data without paying attention to the functions the mobile phone was performing, while three of them were not totally sure. Moreover, of all respondents, $33 \%$ strongly agree and $67 \%$ agree that the collection process was not time consuming because walking is their daily activity. The result on experience indicates that most respondents $(89 \%)$ were familiar with the paths. Two-thirds of them (67\%) volunteered to collect data since they are a member of SoNavNet and the paths are within their commuting area. If the data collected is not appropriate or needs to be updated, $78 \%$ of respondents indicated that they would be willing to follow the same path again, while the rest disagreed. Furthermore, $89 \%$ of respondents would be willing to collect additional data, if needed, and would participate in other collaborative mapping projects of SoNavNet in future. All respondents (100\%) indicated that they would recommend their friends to participate in SoNavNet and collect data if needed. The result of the feedback in the second part indicates that: (1) the task of collecting data is not considered extra activity, (2) the collection process is easy and straightforward, and (3) it is very likely that people would volunteer in pedestrian network data collection projects through social networks, such as SoNavNet.

We plan to introduce SoNavNet to other University of Pittsburgh students in the Fall 2009 semester. Initial tests received 7,080 GPS points which cover more than half of the campus area. The testing area was confined to the University of Pittsburgh's main campus which is surrounded with two types of areas: high rise buildings and an open sky. The mobile phone used to collect the walking trajectories was the Android-based "T-Mobile G1". The uploaded GPS trajectories not only contained the estimated coordinate (latitude/longitude) provided by the GPS receiver, but also included GPS accuracy, bearing, altitude, speed, and time of each location, as well as the user's annotations. The collections included ten walking trajectories: seven trajectories around an open sky area and three trajectories around urban canyons or high rise buildings. For these tests, the walking distance per trajectory was approximately 1600 meters and the average speed was around $1.5 \mathrm{~m} / \mathrm{s}$.

The raw GPS data received from the GPS-enabled mobile phone needs to be analyzed since collecting quality data is essential in generating the prototype pedestrian network base map. The parameter indicating the quality of raw GPS data is positional accuracy, measured in meters. The higher the accuracy, the better the positioning quality will be. GPS accuracy is susceptible to the multipath problem where the signal from satellites is reflected by objects such as buildings, dense vegetation, tunnels, and large vehicles. Generally, pedestrian paths are closer to buildings than roads are, and buildings constitute the main source of interference with GPS signals in urban environments. Therefore, GPS accuracy might be degraded while walking along pedestrian paths next to high buildings. Moreover, because GPS satellites' positions are constantly shifting, data recorded along the same path at different times may yield different accuracies depending on the geometric arrangement of visible satellites.

Table II shows an overall comparison of sky visibility between two locations. The difference between the two locations is the width of the street as well as the environment. Location 1 is a five-lane street with an open area on the left hand side, while location 2 is a two-lane street surrounded with high rise buildings as shown on the right hand side of the table. Since the high rise buildings around location 2 prevent mobile device's receiver from receiving a direct signal from satellites, the accuracy of GPS data in this area is lower than location 1 which has more of an open sky view.

An analysis of the ten trajectories showed that the average number of GPS points per trajectory is equal to 590 and the highest GPS accuracy is $2 \mathrm{~m}$. The average GPS accuracy in the open sky area is about $7.80 \mathrm{~m}$, whereas about $16.20 \mathrm{~m}$. in the narrow road surrounded with high rise buildings. Further analysis reveals that the accuracy of GPS while walking at low speeds was decreased in four notable situations: (1) at the starting point because of time to first fix (TTFF) problem, (2) while stopped, e.g., waiting for the walking signal at a crosswalk, (3) while walking close to high rise buildings, and (4) while walking on a pedestrian covered bridge. However, despite some of these problems, the GPS trajectories collected are useful for constructing pedestrian networks.

The challenges for the next step, editing, are (1) dealing with uncertainty of GPS signal quality, (2) how to combine these raw GPS trajectories to represent a single pedestrian

TABLE II

QUALITY OF GPS DATA IN DIFFERENT AREAS

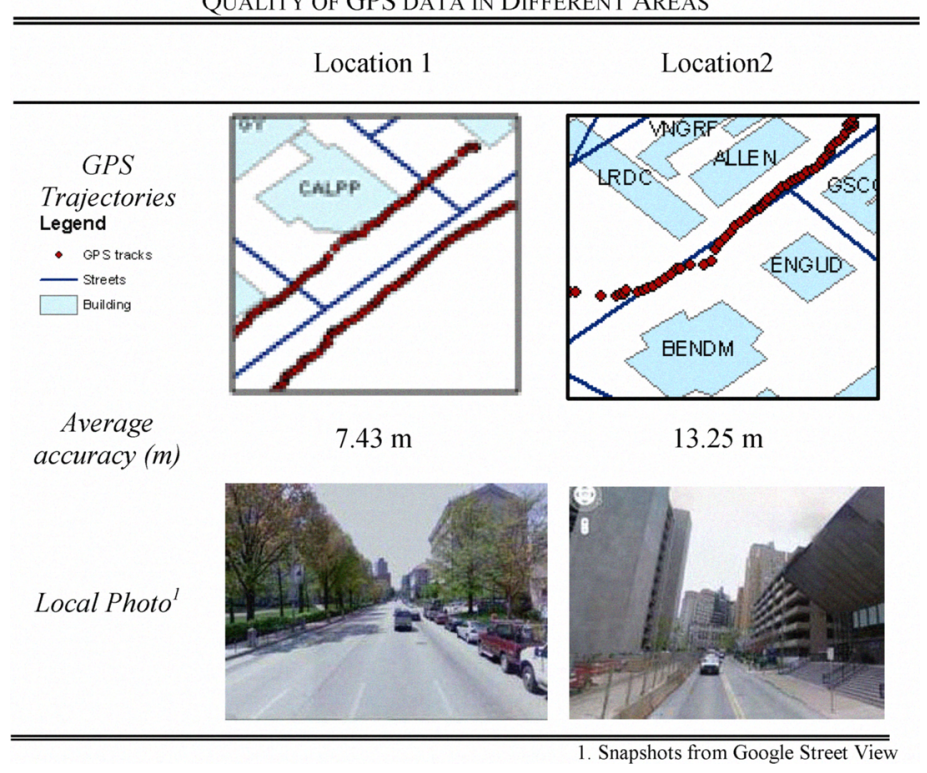



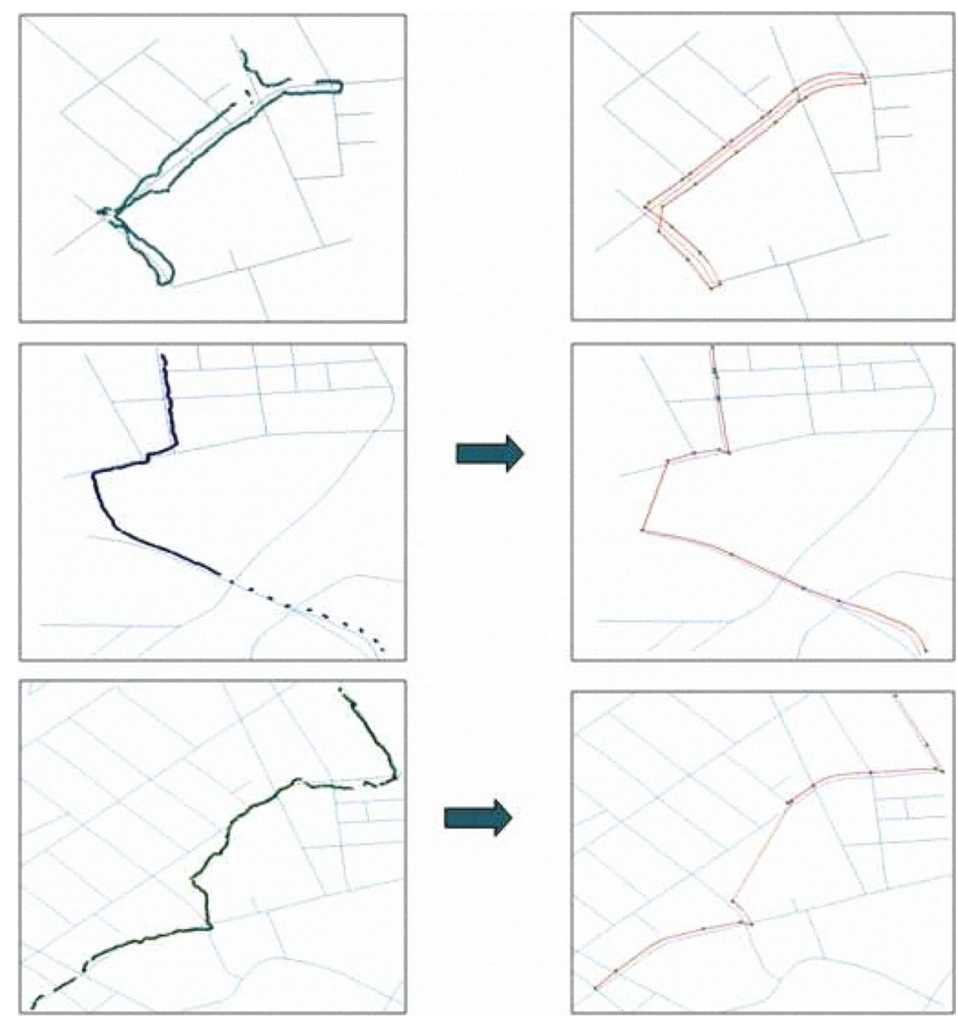

Fig. 4. Examples of constructed pedestrian paths

base map, (3) ensuring the path connectivity, and (4) assuring the geometrical and topological requirement criteria such as completeness, correctness, and quality. An initial solution to these issues consists of four steps: pre-processing, paths determination, paths generation, and paths connectivity. The pre-processing step is to eliminate those data that have significantly different accuracy from other points. Then an algorithm to determine pedestrian path types such as sidewalk, or crosswalk from collected data, is employed. The next step is to generate geometry of each pedestrian path feature and the last step is to construct paths connectivity in a pedestrian network. While the discussion of the methodology and the algorithm for this step is beyond the scope of this paper, we provide examples of pedestrian paths generated from raw GPS data as shown in Fig. 4. The images on the left side are raw GPS trajectories (input) and the images on right side are the vectorized pedestrian paths (output).

\section{CONCLUSIONS AND FUTURE RESEARCH}

This study discussed pedestrian path components with their relations to a pedestrian network and our proposed LBSN approach for pedestrian network data collection. The pedestrian network is an essential resource in a variety of applications, especially pedestrian navigation and urban planning. Alternative methods to generate pedestrian networks are deemed necessary. In this paper, we proposed a collaborative mapping methodology for pedestrian network data collection and dissemination through a LBSN framework called SoNavNet. Collaborative mapping generally consists of four steps starting with collection and ending with distribution. We focused only on the collection of pedestrian base map step in this paper. The collection step allows members to collect GPS trajectories and upload them to an online community for sharing. Although a large number of members is needed to travel pedestrian paths to record trajectories, it is anticipated that, due to the trends of LBSN and GPS-enabled mobile phones, there will be a large pool of volunteers. A prototype of the methodology was developed and tested by nine members of SoNavNet. The feedback on the prototype indicates that it is very likely that people would volunteer in pedestrian network data collection. In addition, the ten trajectories collected both around open sky and in urban canyons are of quality useful for constructing a pedestrian network. In our future research, we plan to develop an algorithm to automatically collect pedestrian paths and to create a base map from raw GPS trajectories.

\section{ACKNOWLEDGMENT}

The authors wish to thank Alper Ozcelik for his assistance in developing SoNavNet. We are also grateful for the assistance of the students in the Geoinformatics Laboratory, University of Pittsburgh, in collecting data and testing SoNavNet.

\section{REFERENCES}

Y. Theodoridis, "Ten Benchmark Queries for Location-Based Services.," The Computer Journal, vol. 46, pp. 713-725, 2003. 
Mobiles: Technologies and Standards," in IEEE International Conference on Communication (ICC'08), Beijing, China, 2008.

[3] P. Kasemsuppakorn and H. A. Karimi, "Data Requirements and a Spatial Database for Personalized Wheelchair Navigation " presented at the 2nd International Convention on Rehabilitation Engineering \& Assistive Technology, Bangkok, Thailand, 2008.

[4] S. Steiniger, M. Neun, and A. Edwardes, "Lecture notes: Foundations of location based services," University of zurich, 2006.

[5] C. Gaisbauer and A. U. Frank, "Wayfinding Model for Pedestrian Navigation," in 11th International Conference on Geographic Information Science University of Girona, Spain, 2008, pp. 1-9.

[6] B. Elias, "Pedestrian Navigation-Creating a tailored geodatabase for routing," in 4th workshop on positioning, navigation and communication (WPNC'07), Hannover, Germany, 2007, pp. 41-47.

[7] M. Hampe and B. Elias, "Integrating topographic information and landmarks for mobile navigation," 2003.

[8] L. Beale, K. Field, D. Briggs, P. Picton, and H. Matthews, "Mapping for Wheelchair Users: Route Navigation in Urban Spaces," The Cartographic journal, vol. 43, pp. 68-81, 2006.

[9] P. Kasemsuppakorn and H. A. Karimi, "Personalised routing for wheelchair navigation," Journal of Location Based Services, vol. 3, pp. 24-54, 2009.

[10] B. Pressel and M. Weiser, "A Computer-Based Navigation System Tailored to the Needs of Blind People," in Computers Helping People with Special Needs. vol. 4061, K. Miesenberger, et al., Eds., ed: Springler Berlin, 2006, pp. 1280-1286.

[11] A. D. Sobek and H. J. Miller, "U-Access: a web-based system for routing pedestrians of differing abilities," Journal of Geographical Systems, vol. 8, pp. 269-287, 2006.

[12] G. K. W. Chin, K. P. V. Niel, B. Giles-Corti, and M. Knuiman, "Accessibility and connectivity in physically activity studies: The impact of missing pedestrian data," Journal of Preventive Medicine, vol. 46, pp. 41-45, 2008.

[13] S. Murugesan, "Understanding Web 2.0," IT Professional, vol. 9, pp. 34-41, 2007.

[14] E. M. Gillavry, "Collaborative Mapping and GIS: An alternative Geographic Information Framework," in Collaborative Geographic Information Systems, S.Balram and S.Dragicevic, Eds., ed: IGI Publishing, 2006, pp. 103-120.

[15] H. A. Karimi, B. Zimmerman, A. Ozcelik, and D. Roongpiboonsopit, "SoNavNet: A Framework for Social Navigation Networks," in International Workshop on Location Based Social Networks (LBSN'09), Seattle, WA, 2009.
[16] WashingtonStateLegislature. (2003, July 5). $R C W \quad 46.04 .400$ Pedestrian. Available:

http://apps.leg.wa.gov/RCW/default.aspx?cite $=46.04 .400$

[17] M. Haklay and P. Weber, "OpenStreetMap: user-generated Street Maps," IEEE Pervasive Computing, vol. 7, pp. 12-18, 2008.

[18] S. Edelkamp and S. Schrodl, "Route planning and map inference with global positioning traces," in Computer Science in perspective, R. Klein, et al., Eds., ed: Berlin: Springer, 2003, pp. 128-151.

[19] R. Bruntrup, S. Edelkamp, S. Jabbar, and B. Scholz, "Incremental map generation with gps traces," in Intelligent transportation systems (ITSC), 2005, pp. 574-579.

[20] K. Laasonen, M. Raento, and H. Toirvonen, "Adaptive On-Device Location Recognition," in Proceedings of Pervasive, 2004, pp. 287-304.

[21] Shyhook. (2009, July 9). WPS (Wi-Fi Positioning System). Available: http://www.skyhookwireless.com/howitworks/wps.php

[22] Skyhook. (2009, XPS - Combining Location Sources. Available: http://www.skyhookwireless.com/howitworks/

[23] InsideGNSS. (2008) New GPS Standard Positioning System (SPS) Performance Standard. 10.

[24] G. Taylor, G. Blewitt, S. Corbett, A. Car, and D. Steup, "Road reduction filtering for GPS-GIS Navigation," Transactions in GIS, vol. 5, pp. 193-207, 2006.

[25] D. M. Boyd and N. B. Ellison, "Social Network Sites: Definition, History, and Scholarship," Journal of Computer-Mediated Communication, vol. 13, pp. 210-230, 2008.

[26] W. Holden. (2009, August 4). Advertising to Fuel Mobile Social Networking Growth as UGC Revenues Reach \$7.3bn by 2013. Available: http://juniperresearch.com/shop/viewpressrelease.php?pr=108

[27] Z.-B. Dong, G.-J. Song, K.-Q. Xie, and J.-Y. Wang, "An experimental study of large-scale mobile social network," in the 18 th international conference on World wide web, Madrid, Spain 2009, pp. 1175-1176.

[28] B. Antal. (2008, Aug 5). Upcoming Trend: GPS-Enabled Cell Phones.

Available: http://www.devhardware.com/c/a/Opinions/Upcoming-TrendGPSEnabled-Cell-Phones/

[29] L. D. Frank, M. A. Andresen, and T. L. Schmid, "Obesity Relationships with Community Design, Physical Activity, and Time Spent in Cars," American Journal of Preventive Medicine, vol. 27, pp. 89-96, 2004. 